\title{
In silico Investigation and Biological Evaluation of Synthesized Sulfamethoxazole Derivatives
}

\author{
JYOTIRMAYA SAHOO ${ }^{1}$, PRIYAMBADA KSHIRODA, NANDINI SARANGI², S. K. ROUT ${ }^{3}$, AND S. K. PAIDESETTY3* \\ School of Pharmacy, ARKA Jain University, Gamharia, Seraikela, Kharsawan, Jameshedpur, Jharkand-832 108 , \\ ${ }^{1}$ Department of Pharmaceutics, ${ }^{2}$ Department of Pharmaceutical Chemistry, Sri Jayadev College of Pharmaceutical Sciences, \\ Bhubaneswar-752 101, 'Department of Pharmaceutical Chemistry, School of Pharmaceutical Sciences, Siksha 'O' Anusand- \\ han (Deemed to be University) Bhubaneswar-751 030, India
}

Sahoo et al.: In silico investigation and biological evaluation of Sulfamethoxazole derivatives

\begin{abstract}
A series of 4-[4-(substitutedaryl/heteroaryldiazenyl]-N-(5-methylisoxazol-3-yl)benzene sulphonamide derivatives (4a-4f) were designed and synthesized coupling a mixture of diazotized sulfamethoxazole with six different phenolic and enolic compounds in an in situ reaction. The structural environment of synthesis of each molecule was confirmed by Fourier-transform infrared spectroscopy, proton nuclear magnetic resonance and elemental analysis. These derivatives were further screened in various biological assays in vivo for analgesic and antiinflammatory activities and in vitro for antioxidant and antimicrobial activities. When tested for analgesic activity at a dose of $50 \mathrm{mg} / \mathrm{kg}$, compounds 4-((2-hydroxynaphthalen1-yl)diazenyl)-N-(5-methylisoxazol-3-yl)benzene sulphonamide (4d) and 4-((4-hydroxy-5-isopropyl-2methylphenyl)diazenyl)-N-(5-methylisoxazol-3- yl)benzene sulphonamide (4f) showed 58.33 and $57.76 \%$ of pain inhibition, respectively. These two molecules also exhibited significant antioxidant activity at 10 and $50 \mu \mathrm{g} / \mu \mathrm{l}$. The compound 4-[(4-hydroxy-2-oxo-2H-chromen-3-yl)diazenyl]-N-(5-methylisoxazol3-yl)benzene sulphonamide (4a) exhibited antibacterial activity against Staphylococcus aureus resistance, Candida albicans and DescriptionCryptococcus neoformans at a concentration of $31.25 \mu \mathrm{g} / \mathrm{ml}$. The analgesic action of these synthesised analogues was predicted in molecular docking experiments with a specific target protein, cyclooxgenase-2 of Mus musculus and results indicated all tested compounds to exhibit good binding interaction with the active site amino acid of the target enzyme.
\end{abstract}

Key words: Sulfanilamide, thymol, 4-hydroxy coumarin, antioxidant, antimicrobial, antiinflammatory

Sulfanilamides were established molecules in the field of medicine. Though these are rarely prescribed these days, but in medicinal chemistry the importance of sulfanilamide entity is well-recognised as it continues to offer several therapeutic benefits for drug development ${ }^{[1]}$. Some $70-80$ y ago, a red dye Prontosil, an azo-linked sulfonamide pro-drug was widely used to treat streptococcal infections ${ }^{[2]}$. For antibacterial drug development identification of correct lead candidates is a major challenge. Thus, sulfanilamide analogues continue to offer insights for the development of newer antimicrobials.

Isoxazole is a heterocyclic azole moiety with oxygen and nitrogen atoms in cyclopentadiene ring. Compounds bearing the isoxazole ring serve as an important source for developing useful drug candidates, for treating several infectious diseases. Although, majority of isoxazole derivatives have exhibited

*Address for correspondence

E-mail: sairampaidesetty@gmail.com

January-February 2020 immunosuppressant and antiinflammatory activities, sulfonamide containing isoxazole analogues were found to display potent analgesic and antiinflammatory action. The commercially available isoxazole bearing drugs are COX-2 inhibitor, valdecoxib, leflunomide, dihydrofolate synthetase inhibitor, sulfamethoxazole (SMZ), sulfisofurazole and $\beta$-lactamase-resistant antibiotics, such as cloxacillin and dicloxacillin to mention some.

Moreover, literature revealed that molecules with a diazenyl $(-\mathrm{N}=\mathrm{N}-)$ group showed versatile biological properties ${ }^{[3]}$ and also that nitrogen bearing heterocyclic

This is an open access article distributed under the terms of the Creative Commons Attribution-NonCommercial-ShareAlike 3.0 License, which allows others to remix, tweak, and build upon the work non-commercially, as long as the author is credited and the new creations are licensed under the identical terms

Accepted 02 December 2019

Revised 18 September 2019

Received 08 Juy 2019

Indian J Pharm Sci 2020;82(1):123-130 
compounds possess good biological action ${ }^{[4]}$. Microorganisms cause infectious disease and due to irrational use of antibiotics, some of the pathogenic organisms have developed resistance against these antimicrobial drugs. Thus, Research efforts are to be focused more towards developing drugs that act against the resistant pathogenic organisms with minimal side effects to create socio-economic benefit. Cellular oxidative stress in biological system due to generation of reactive oxygen species (ROS) leads to alterations in genes through oxidation of nucleic acid, impairment of muscle function by protein denaturation, while lipid peroxidation in cells cause perturbation of homeostasis resulting in cell death. ROS are produced during tissue injury and antioxidants play a vital role during wound healing. Although, inflammation is a defensive mechanism in response to foreign bodies but leads to cellular damage due to producing oxidative stress ${ }^{[5]}$. Under these circumstances, synthetic and natural antioxidants have a major role to protect cells against oxidative stress $^{[6]}$. Hence, it is obligatory to develop new drugs at minimum cost, which are effective against resistant organisms and also aid in quick healing of infected wounds through free radical scavenging ${ }^{[7]}$.

Previous studies from our laboratory explored the synthesis and characterisation of phenolic/enolic substituted diazenyl SMZ derivatives ${ }^{[8-14]}$. To continue these efforts, biological activities of these analogues needs to be evaluated using different models. The present work is conceptualized on the basis of literature to design some hybrid molecules containing sulfonamide, 5-methyl-isoxazole moiety and diazenyl group all together with different phenolic/enolic systems with in these structures. In this drug design attempt it was planned to couple 6 bioactive neutral nucleophiles such as, 4-hydroxy coumarin (4-HC), 8-hydroxy quinoline (8-HQ), salicylic acid, $\beta$-napthol, salicylaldehyde and thymol with diazonium salt of SMZ to produce the desired molecules $4 \mathrm{a}-4 \mathrm{f}$ to evaluate the biological actions possessed by these molecules. In addition, molecular docking study was carried out to predict the potential of the molecules to exhibit analgesic and antiinflammatory activities. Thus, all designed molecules were subjected to molecular docking against the cyclooxygenase (COX-2) enzyme, the structure of which was retrieved from the Protein Data Bank.

\section{MATERIALS AND METHODS}

All chemicals (Merck Specialties Ltd., and Hi-Media Laboratories Pvt. Ltd., Mumbai, India) used were of synthetic and analytical grade. Melting points $\left(^{\circ}\right)$ were determined using the open capillary method (Elico) and were uncorrected. The IR spectra, molecular mass, ${ }^{1} \mathrm{HNMR}$ spectra and elemental analysis were carried out on Jasco FT/IR 4100, Japan, Shimadzu, Bruker ${ }^{1} \mathrm{H}$ NMR, $400 \mathrm{MHz}$ and Perkin Elmer 2400, respectively. The UV absorption $\left(\lambda_{\max }\right)$ maximum was measured on a Jasco V-630 spectrophotometer

\section{In silico investigation:}

COX-2 of Mus musculus with PDB ID: 1CX2 was retrieved from Protein Data Bank (PDB) and docked against the proposed molecules with Arugus Lab 4.0 individually. The protein-ligand interaction was carried out by Discovery Studio Visualizer 3.1 software.

\section{Synthesis of the proposed molecules (4a-4f):}

The synthetic procedures of compounds (4a-4f) have been previously reported (fig. 1) ) $^{[8-12]}$. A cold solution of sodium nitrite was added dropwise to an aqueous solution of the desired SMZ with concentrated hydrochloric acid; the temperature of the reaction mixture was maintained at $0-5^{\circ}$. When addition was completed, the solution was to stand a few minutes with occasional stirring to complete diazotization. Then it was poured into an ice cold solution of individual phenolic/enolic compounds (2a-2f) in $20 \%$ sodium hydroxide the reaction mixture was stirred and kept overnight in a refrigerator. The final precipitate obtained was filtered and recrystallized from hot ethanol.

\section{Spectral characterization:}

The spectral data of individual analogues $4 \mathrm{a}-4 \mathrm{f}$ was previously reported. 4-[(4-hydroxy-2-oxo- $2 \mathrm{H}$ chromen-3-yl)diazenyl]- $N$-(5-methylisoxazol-3-yl) benzene sulphonamide (4a)- red colour; yield, $72 \%$; $\mathrm{R}_{\mathrm{f}}$; $0.8, \mathrm{mp}\left(^{\circ}\right) ; 225-228$; UV/Vis $\left(\lambda_{\max }, \mathrm{nm}, \mathrm{CH}_{3} \mathrm{OH}\right): 403$; IR (KBr, $\left.\gamma, \mathrm{cm}^{-1}\right)$ : 3179 (O-H str.), $1726(\mathrm{C}=\mathrm{O}$ str. $), 1617$ $(-\mathrm{C}=\mathrm{C}-$ str. $), 2925\left(\mathrm{CH}_{2}\right.$ str. $), 1506(-\mathrm{N}=\mathrm{N}-), 1390,1157$ $\left(\mathrm{SO}_{2}\right.$ str.); ${ }^{1} \mathrm{HNMR}\left(\mathrm{CDCl}_{3} \delta \mathrm{ppm}, 400 \mathrm{MHZ}\right): 2.43$ (s, $\left.3 \mathrm{H}, \mathrm{CH}_{3}\right), 7.93$ (d, coumarin $\left.\mathrm{H}-5\right), 7.654$ (m, coumarin H-6), 7.65 (m, coumarin H-7), 7.42 (d, coumarin H-8), 5.62 (s, 1H, isoxazol-4-yl H); LC-MS (\% area); 100; $\mathrm{m} / \mathrm{z} ; 427.18(\mathrm{M}+1)$; analysis for $\mathrm{C}_{19} \mathrm{H}_{13} \mathrm{~N}_{4} \mathrm{O}$ S: Calcd \% C, 53.52; H, 3.31; N, 13.14;S,7.52; found \%: C, 53.47; $\mathrm{H}, 3.36 ; \mathrm{N}, 13.21 ; \mathrm{S}, 7.49$.

4 -((8-hydroxyquinolin-5-yl)diazenyl)- $N$ - (5methylisoxazol-3-yl)benzene sulphonamide (4b)orange colour; yield $92 \%$; $\mathrm{R}_{\mathrm{f}}$; 0.8, mp $\left(^{\circ}\right) ; 220-223$; $\mathrm{UV} / \mathrm{Vis}\left(\lambda_{\max }, \mathrm{nm}, \mathrm{CH}_{3} \mathrm{OH}\right): 403$; IR $\left(\mathrm{KBr}, \gamma, \mathrm{cm}^{-1}\right)$ : 3297 (O-H str.), 1616 (-C=C- str.), $2847\left(\mathrm{CH}_{2}\right.$ str.), 


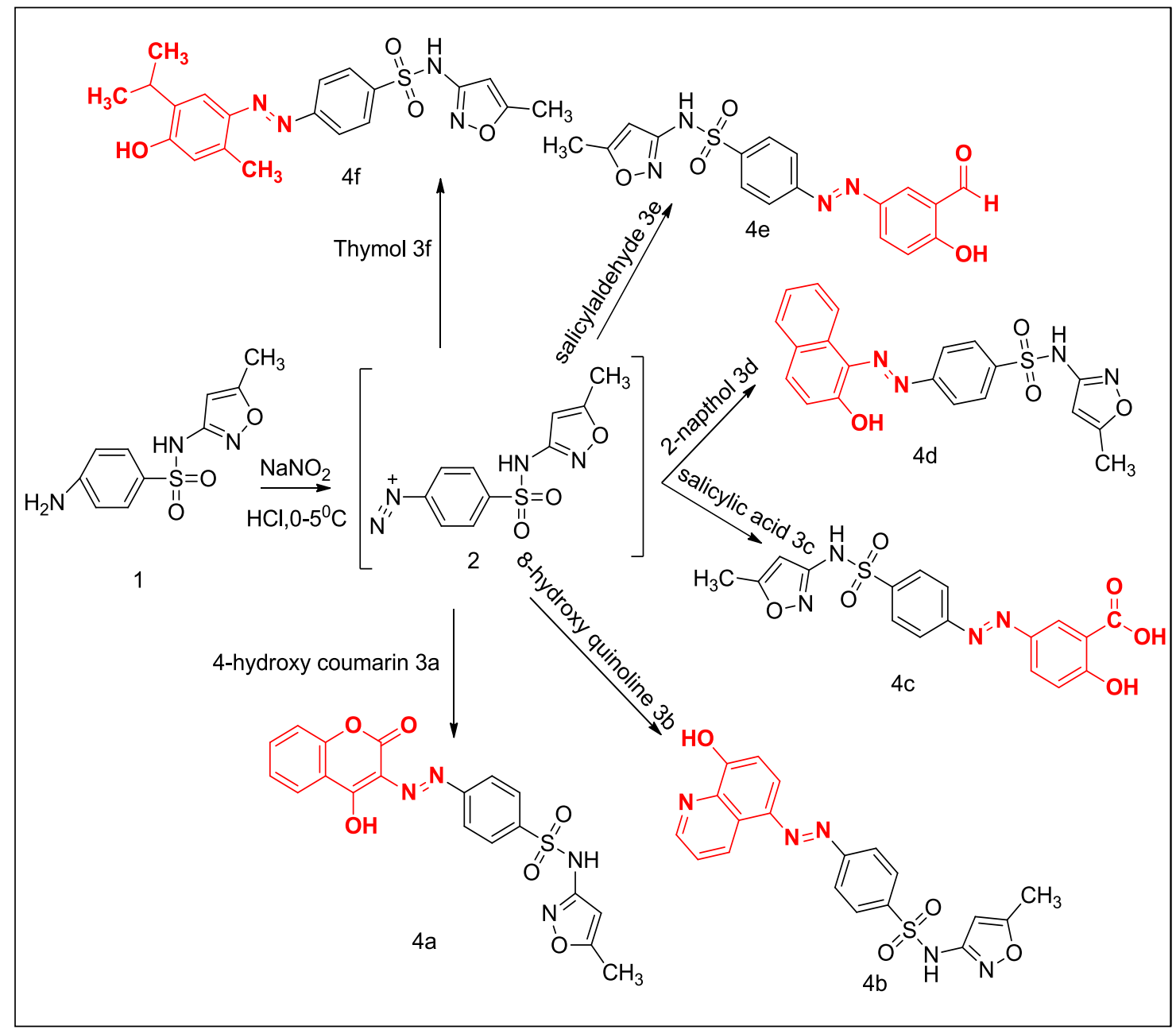

Fig. 1: Synthesis of sulfamethoxazole analogues $4 a-4 f$

1573 (C=N str.), 1506 (-N=N-), $13411170\left(\mathrm{SO}_{2}\right.$ str.); ${ }^{1} \mathrm{HNMR} \quad\left(\mathrm{DMSO}-d_{6}, \delta\right.$ ppm, 400 MHZ): 2.83 $\left(\mathrm{s}, 3 \mathrm{H}, \mathrm{CH}_{3}\right), 8.01-8.05$ (m,4H,ArH), 9.28 (d, quinolinyl $\mathrm{H}-2), 7.63$ (d, quinolinyl H-3), 8.03 (d, quinolinyl H-4), 8.07 (d, quinolinyl H-6), 7.26 (d, quinolinyl H-7), 6.28 (s,1H,isoxazol-4-yl H); LC-MS (\% area); 100; $\mathrm{m} / \mathrm{z}$; $410.30(\mathrm{M}+1)$; Analysis for $\mathrm{C}_{19} \mathrm{H}_{15} \mathrm{~N}_{5} \mathrm{O}_{4} \mathrm{~S}$ : Calcd \% C, 55.74; H, 3.69; N, 17.11 S, 7.67; found \%: C, 55.72; H, $3.73 ; \mathrm{N}, 17.13 ; \mathrm{S}, 7.85$.

2-hydroxy-5-((4-(N-(5-methylisoxazol-3-yl) sulfamoyl)phenyl)diazenyl)benzoic acid (4c)- Grey colour powder; yield $73 \%$; $\mathrm{R}_{\mathrm{f}}$; 0.8, mp ( $\left.{ }^{\circ}\right)$; 227-230; UV/Vis $\left(\lambda_{\max }, \mathrm{nm}, \mathrm{C}_{2} \mathrm{H}_{5} \mathrm{OH}\right): 370$; IR $\left(\mathrm{KBr}, \gamma, \mathrm{cm}^{-1}\right)$ : 3461 (N-H str.), 1668 (C=O str.), 1614 (-C=C- str.), $2922\left(\mathrm{CH}_{2}\right.$ str. $), 1573$, (C=N str.), 1510 (-N=N-), 1315, 1170 ( $\mathrm{SO}_{2}$ str.); ${ }^{1} \mathrm{HNMR}$ (DMSO- $d_{6}, \delta \mathrm{ppm}, 400 \mathrm{MHZ}$ ): 7.25-8.01 (m,7H,ArH), 2.43 (s, 3H, $\left.\mathrm{CH}_{3}\right), 11.67$ (s, IH, $\mathrm{OH}), 12,11(\mathrm{sb}, 1 \mathrm{H}, \mathrm{COOH}), 6.17(\mathrm{~s}, 1 \mathrm{H}$, isoxazol-4-yl $\mathrm{H})$; LC-MS (\% area); 100; $\mathrm{m} / z$; $403.18(\mathrm{M}+1)$; analysis for $\mathrm{C}_{17} \mathrm{H}_{14} \mathrm{~N}_{4} \mathrm{O}_{6} \mathrm{~S}$ : Calcd \% C, 50.75; H, 3.51; N, 13.92; S,7.97; found \%: C, 50.47; H, 3.55; N, 13.96; S,7.89. 4-((2-hydroxynaphthalen-1-yl)diazenyl)- $N$-(5methylisoxazol-3-yl)benzene sulfonamide (4d)-Orange red colour powder; yield $90 \%$; $\mathrm{R}_{\mathrm{f}} ; 0.8, \mathrm{mp}\left(^{\circ}\right)$; 170178; UV/Vis ( $\left.\lambda \max , \mathrm{nm}, \mathrm{C}_{2} \mathrm{H}_{5} \mathrm{OH}\right): 470$; IR $(\mathrm{KBr}, \gamma$, $\left.\mathrm{cm}^{-1}\right)$ : 3251 (O-H/NH str.), 1511, 1607 (C=C str.),1460 $(-\mathrm{N}=\mathrm{N}-), 1385$ (O-H bend.), 1316, 1159 ( $\mathrm{SO}_{2}$ str.), 1212 (C-O str.), 956 (S-N str.); ${ }^{1} \mathrm{H} \mathrm{NMR}\left(\mathrm{CDCl}_{3}, \delta\right.$ ppm, 400 MHZ): 17.49 (s, 1H, SO $\mathrm{SH}_{2}, 16.00(1 \mathrm{H}, \mathrm{OH}), 7.85-$ 8.34 (m, 4H, ArH), 7.14-7.85 (6H, naphthyl H), 6.26 (s, 1H. isoxazol-4yl H), 2.37 (s, 3H, $\left.\mathrm{CH}_{3}\right)$; LC-MS (\% area); $97 ; m / z ; 409.68(\mathrm{M}+1)$; analysis for $\mathrm{C}_{20} \mathrm{H}_{16} \mathrm{~N}_{4} \mathrm{O}_{4} \mathrm{~S}$ : Calcd \% C, 58.84; H, 3.97; N, 13.71; S, 7.86; found \%: C, 50.81; H, 3.95; N, 13.72; S, 7.85.

4-((3-formyl-4-hydroxyphenyl) diazenyl)- $N$-(5methylisoxazol-3-yl) benzene sulfonamide (4e)- Grey colour powder; yield $90 \%$; $\mathrm{R}_{\mathrm{f}} ; 0.8, \mathrm{mp}\left(^{\circ}\right)$; 170-175; UV/Vis $\left(\lambda_{\max }, \mathrm{nm}, \mathrm{C}_{2} \mathrm{H}_{5} \mathrm{OH}\right): 450$; IR $\left(\mathrm{KBr}, \gamma, \mathrm{cm}^{-1}\right)$ : 3442 (O-H str.), 2915 (CH str.), 1660 (C=O str.), 1615 $(\mathrm{C}=\mathrm{C}$ str. $), 1478(-\mathrm{N}=\mathrm{N}-), 1305,1175\left(\mathrm{SO}_{2}\right.$ str. $), 1137$ (C-O str.), 901 (S-N str.); ${ }^{1} \mathrm{HNMR}$ (DMSO- $d_{6,} \delta$ ppm, $400 \mathrm{MHZ}$ ): 11.42 (s, 1H, OH), 11.02 (s, 1H, SO $\mathrm{S}_{2} \mathrm{NH}$ ), 
10.03 (s, 1H, CHO), 8.18-8.23 (m, 4H, Ar H), 8.01 (d, 1H, salicylaldehyde $\mathrm{H}-6), 7.98$ (s, $1 \mathrm{H}$, salicylaldehyde $\mathrm{H}-2), 7.53$ (d, 1H, salicylaldehyde H-5), 6.24 (s, 1H, isoxazole $\mathrm{H}-4), 2.38\left(\mathrm{~s}, 3 \mathrm{H},=\mathrm{C}-\mathrm{CH}_{3}\right)$; LC-MS (\% area); $100 ; \mathrm{m} / \mathrm{z} ; 386.22(\mathrm{M}+1)$; analysis for $\mathrm{C}_{17} \mathrm{H}_{14} \mathrm{~N}_{4} \mathrm{O}_{5} \mathrm{~S}$ : Calcd \% C, 52.83; H, 3.67; N, 14.51;S,8.29; found \%: C, 50.85; H, 3.68; N, 14.52; S, 8.33.

4-((4-hydroxy-5-isopropyl-2-methylphenyl)diazenyl)$N$-(5-methylisoxazol-3-yl)benzene sulphonamide (4f)- Brick red colour powder; yield $84 \%$; $\mathrm{R}_{\mathrm{f}} ; 0.8$, $\mathrm{mp}\left({ }^{\circ}\right)$; 304-310; UV/Vis ( $\lambda \max , \mathrm{nm}, 1,4$-dioxane): 409; IR (KBr, $\left.\gamma, \mathrm{cm}^{-1}\right)$ : 3489, 3163 (O-H str.), 2961 $\left(\mathrm{CH}_{2}\right.$ str. $), 1619$ (C=C str.), $1466(-\mathrm{N}=\mathrm{N}-), 1341,1129$ ( $\mathrm{SO}_{2}$ str.), 899 (S-N str.). ${ }^{1} \mathrm{H}$ NMR (DMSO- $d_{6} \delta$ ppm, $400 \mathrm{MHZ}): 9.75\left(\mathrm{~s}, 1 \mathrm{H}, \mathrm{SO}_{2} \mathrm{NH}\right), 9.56(\mathrm{~s}, 1 \mathrm{H}, \mathrm{OH})$, 7.95-8.50 (m, 4H, Ar H), 7.85 (s, 1H, thymol H-6), 6.95 (s, 1H, thymol $\mathrm{H}-3), 6.80(\mathrm{~s}, 1 \mathrm{H}$, isoxazolyl $\mathrm{H}), 2.97$ $\left(\mathrm{m}, 1 \mathrm{H}, \mathrm{CH}\left(\mathrm{CH}_{3}\right)_{2}\right), 2.38(\mathrm{~s}, 3 \mathrm{H}$, methyl), $1.25(\mathrm{~d}, 6 \mathrm{H}$, $\mathrm{CH}\left(\mathrm{CH}_{3}\right)_{2}$; LC-MS (\% area); 100; $\mathrm{m} / \mathrm{z} ; 415.18(\mathrm{M}+1)$; analysis for $\mathrm{C}_{20} \mathrm{H}_{22} \mathrm{~N}_{4} \mathrm{O}_{4} \mathrm{~S}$ : Calcd \% C, 57.85; H, 5.29; $\mathrm{N}, 13.45 ; \mathrm{S}, 7.68$; found $\%$ : C, 57.96; H, 5.55; N, 13.52; S,7.74 (Table 1, fig. 2)

\section{Pharmacological evaluation:}

Acute oral toxicity of the synthesised molecules was determined using recommended procedure as prior OECD guideline No. 420. The animals were orally administered with the test molecules at the doses of 5, 50,300 and $2000 \mathrm{mg} / \mathrm{kg}$ at an interval of $24 \mathrm{~h}$ between of each dose. The entire study was carried out as per CPCSEA and IAEC guidelines (registration number 1171/C/08/CPCSEA and Ref. No. 60/SPS/IAEC/ SOAU).

In vivo acetic acid-induced writhing method was carried out with a little modification to evaluate the analgesic activity ${ }^{[15]}$. The group I was treated as a negative control and the group II was as positive control and was administered orally $100 \mathrm{mg} / \mathrm{kg}$ acetylsalicylic acid (ASA). Animals from groups IIIXIV were orally administered with test molecules (4a$4 \mathrm{f}$ ) at the dose level of 25 and $50 \mathrm{mg} / \mathrm{kg}$. After $1 \mathrm{~h}$ of the administrations, all the groups were treated with $0.6 \%$ $\mathrm{v} / \mathrm{v}$ acetic acid solution $(1 \mathrm{ml} / 100 \mathrm{~g})$ intra peritoneally and the onset and number of writhings was noted. Finally percent of analgesic activity was calculated as follows, $\%$ analgesic activity $=$ mean writhing count (control-treated group)/mean writhing count of control group $\times 100$. The reaction time was expressed as mean \pm SEM. The statistical analysis was done by one way-ANOVA followed by Dunnett's $t$-test.

Eddy's hot plate (Techno) was used to induce pain by thermal stimuli and to measure the response latencies as per method first described by Eddy and Leimback ${ }^{[13]}$ with a slight modification and the instrument was adjusted to $55 \pm 0.5^{\circ}$ and the basal reaction time of each animal were recorded before administering either the test or standard compounds. The reaction time for all the groups was measured at an interval of $60 \mathrm{~min}$ for $3 \mathrm{~h}$ after administration of test molecules and finally the percent analgesic activity (reaction time) was determined. Carrageenan-induced rat hind paw oedema method was followed with a slight deviation using the Ugo Basile plethysmometer (7150). All treatments were given orally $\mathrm{I} h$ before the injection of carrageenan. Percent inhibition of inflammation was calculated using the formula, $\%$ inhibition $=100(1-\mathrm{Vt} / \mathrm{Vc})$, where $\mathrm{Vc}$ represents oedema volume in control and Vt oedema volume in group treated with test molecules.

\section{In vitro radical scavenging capacity:}

The antioxidant activity of the prepared isoxazole derivatives were measured using the 2,2-diphenyl-

TABLE 1: PHYSICAL CHARACTERISTIC DATA OF SULFAMETHOXAZOLE ANALGUES 4A-4F

\begin{tabular}{|c|c|c|c|c|c|c|c|}
\hline Comps. & Heteroaryl & M. formula & $m / z$ & Rf & $\mathrm{mp}\left({ }^{\circ}\right)$ & Color & $\begin{array}{l}\text { Yield } \\
(\%)\end{array}$ \\
\hline $4 a$ & $\begin{array}{l}\text { 4-[(4-hydroxy-2-oxo-2H-chromen-3-yl)diazenyl]-N- } \\
\text { (5-methylisoxazol-3-yl)benzene sulphonamide }\end{array}$ & $\mathrm{C}_{19} \mathrm{H}_{14} \mathrm{~N}_{4} \mathrm{O}_{6} \mathrm{~S}$ & 427.00 & 0.8 & $260-270$ & $\begin{array}{l}\text { Bright } \\
\text { yellow }\end{array}$ & 75 \\
\hline $4 b$ & $\begin{array}{l}\text { 4-((8-hydroxyquinolin-5-yl) diazenyl) }-N \text { - (5-methyl } \\
\text { isoxazol-3-yl) benzene Sulphonamide }\end{array}$ & $\mathrm{C}_{19} \mathrm{H}_{15} \mathrm{~N}_{5} \mathrm{O}_{4} \mathrm{~S}$ & 410.30 & 0.8 & $220-223$ & $\begin{array}{l}\text { Brick } \\
\text { red }\end{array}$ & 92 \\
\hline $4 c$ & $\begin{array}{c}\text { 2-hydroxy-5- } \\
((4-(N-(5-m e t h y l i s o x a z o l-3-y l) \\
\text { sulfamoyl) phenyl) diazenyl }) \\
\text { benzoic acid }\end{array}$ & $\mathrm{C}_{17} \mathrm{H}_{14} \mathrm{~N}_{4} \mathrm{O}_{6} \mathrm{~S}$ & 403. 00 & 0.8 & $227-230$ & Grey & 73 \\
\hline $4 d$ & $\begin{array}{l}\text { 4-((2-hydroxynaphthalen-1-yl)diazenyl)- } N-(5- \\
\text { methylisoxazol-3-yl) benzene sulphonamide }\end{array}$ & $\mathrm{C}_{20} \mathrm{H}_{16} \mathrm{~N}_{4} \mathrm{O}_{4} \mathrm{~S}$ & 409.30 & 0.8 & $170-174$ & $\begin{array}{l}\text { Orange } \\
\text { red }\end{array}$ & 90 \\
\hline $4 e$ & $\begin{array}{l}\text { 4-((3-formyl-4-hydroxyphenyl) diazenyl)- } N-(5- \\
\text { methylisoxazol-3-yl) benzene sulfonamide }\end{array}$ & $\mathrm{C}_{17} \mathrm{H}_{14} \mathrm{~N}_{4} \mathrm{O}_{5} \mathrm{~S}$ & 386.22 & 0.8 & $170-172$ & Grey & 90 \\
\hline $4 f$ & $\begin{array}{l}\text { 4-((4-hydroxy-5-isopropyl-2-methylphenyl)diazenyl)- } \\
\text { N-(5-methylisoxazol-3-yl)benzenesulphonamide }\end{array}$ & $\mathrm{C}_{20} \mathrm{H}_{22} \mathrm{~N}_{4} \mathrm{O}_{4} \mathrm{~S}$ & 414.14 & 0.8 & $304-310$ & $\begin{array}{l}\text { Brick } \\
\text { red }\end{array}$ & 84 \\
\hline
\end{tabular}




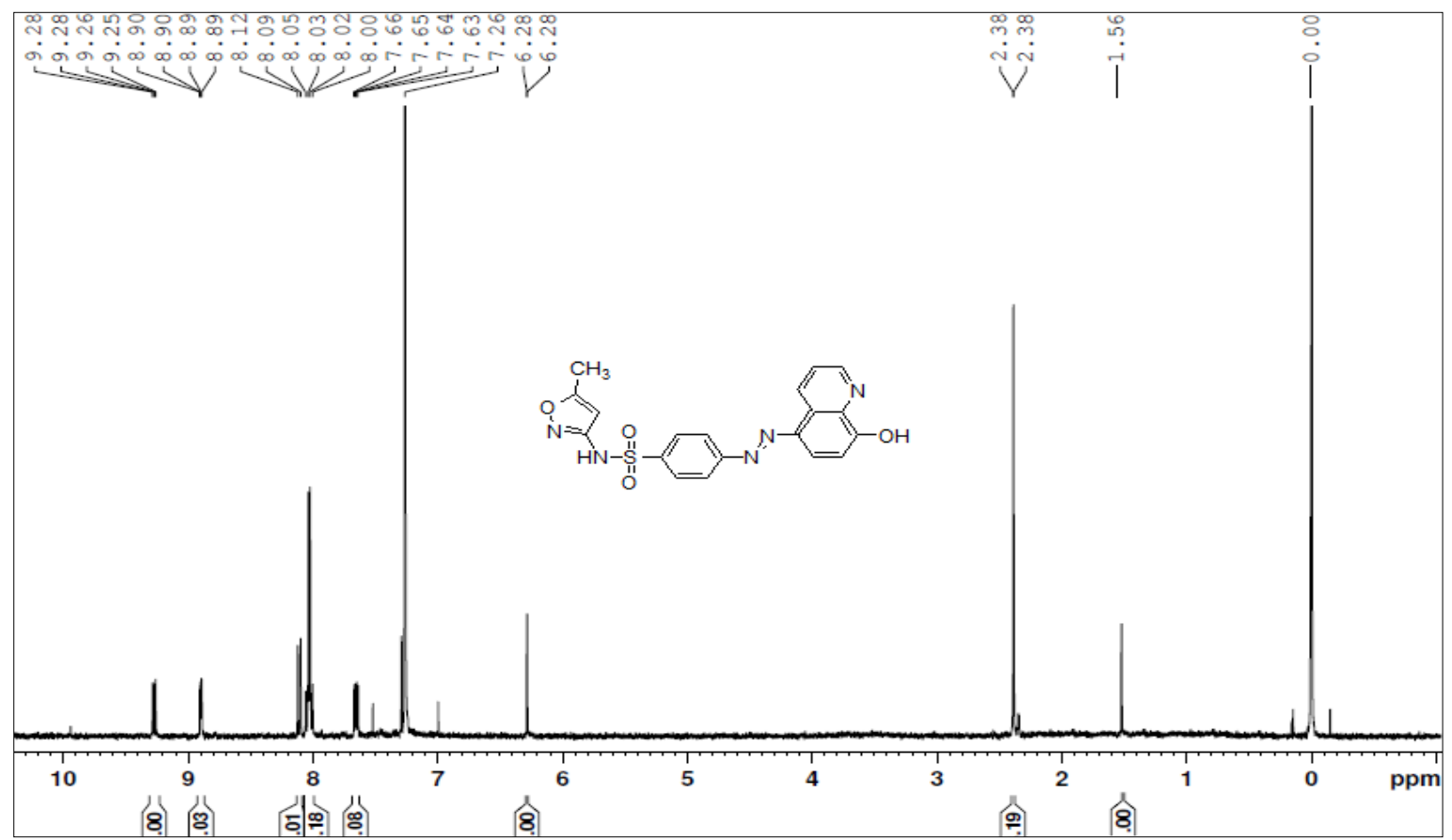

Fig. 2: ${ }^{1} \mathrm{HNMR}$ of 4-((8-hydroxyquinolin-5-yl)diazenyl)-N-(5-methylisoxazol-3-yl)benzene sulfonamide (4b)

1-picrylhydrazyl (DPPH) assay procedure with some modifications ${ }^{[6]}$ at several concentrations, the absorbance of the test molecules and the standard butylated hydroxy toluene (BHT) was measured at $517 \mathrm{~nm}$ on a UV/Vis spectrophotometer and the antioxidant activity was calculated. A mixture of DPPH and methanol was considered as control. All experiments were carried out in triplicate. Followed by Dunnett's post hoc test, the $\mathrm{IC}_{50}$ values were expressed as mean $\pm \mathrm{SD}$. Inhibition (\%) $=\left[\left(\mathrm{A}_{\text {cont }}-\mathrm{A}_{\text {test }}\right) / \mathrm{A}_{\text {test }}\right] \times 100, \mathrm{~A}_{\text {cont }}=$ absorbance of control, $\mathrm{A}_{\text {test }}=$ absorbance of the test and standard samples, The $\mathrm{IC}_{50}$ value was graphically determined.

\section{Microbiological evaluation:}

The antimicrobial activity of the synthesized molecules was investigated according to the agar well diffusion method using the nutrient agar and Sabouraud dextrose agar medium (HI-Media) for bacteria and fungi, respectively. The antimicrobial diffusion test was performed using a cell suspension of about $1.5 \times 10^{6}$ $\mathrm{CFU} / \mathrm{ml}$ employing a McFarland turbidity standard No. $0.5^{[14]}$. The microbial strains $K$. pneumonia (MTCC 109) Candida albicans (MTCC 3017) and C. neoformans (MTCC 3019) were procured from the Institute of Microbial Technology and Gene bank (IMTECH), Chandigarh, India. Escherichia coli and Staphylococcus aureus resistant to norfloxacin, ofloxacin, and ampicillin were isolated from the urine sample collected from UTI patients at the IMS, SUM Hospital, Bhubaneswar, India. Amoxicillin and fluconazole was used as reference standards against bacterial and fungal strains, respectively. The lowest concentration of the test compounds inhibiting visible growth for bacteria and fungi was termed as minimum inhibitory concentration (MIC). Test solutions of synthesized molecules were prepared suing the twofold dilution method at a concentration level ranging from $500-31.25 \mu \mathrm{g} / \mathrm{ml}$ using DMF to evaluate the MIC.

\section{Statistical analysis:}

Dunnett's post hoc test and Dunnett's $t$-test were used and the observed data subjected to one way analysis of variance (ANOVA).

\section{RESULTS AND DISCUSSION}

A series of phenolic/enolic substituted azosulfamethoxazoles $4 \mathrm{a}-4 \mathrm{f}$ were synthesized by the in situ reactions between diazotised SMZ and 6 different individual phenolic and enolic compound under mild conditions. All the proposed isoxazole derivatives were synthesized, physical properties and spectral interpretation were made (Table 1), which were similar to those reported earlier ${ }^{[8-12]}$.

The research work was aimed to theoretically validate the binding of the synthesized SMZ derivatives (4a-4f) using molecular docking against COX-2 protein of Mus musculus as depicted in Table 2. The docking score and molecule interactions (fig. 3) were obtained in least binding energy of the compound $4 \mathrm{~d}$ and $4 \mathrm{f}$ at value -12.980 and $-12.386 \mathrm{kcal} / \mathrm{mol}$ with 
TABLE 2: CONVERTED 3D- STRUCTURES AND DOCKING SCORES OF INDIVIDUAL SMZ ANALGUES 4A4F AGAINST TARGET ENZYME CYCLOOXYGENASE-2

\begin{tabular}{|c|c|c|c|c|}
\hline Comps. & Chemical name & 3D- structure & $\begin{array}{l}\text { Docking } \\
\text { score (kcal/ } \\
\text { mol) }\end{array}$ & $\begin{array}{l}\text { Interaction with amino acid of } \\
\text { target enzyme during docking }\end{array}$ \\
\hline $4 a$ & $\begin{array}{l}\text { 4-[(4-hydroxy-2-oxo-2H- } \\
\text { chromen-3-yl)diazenyl]-N- } \\
\text { (5-methylisoxazol-3-yl) } \\
\text { benzene sulphonamide }\end{array}$ & & -10.958 & $\begin{array}{l}\text { LYS83, PRO8, VAL89, LEU93, } \\
\text { ILE112, TYR115, TYR122, PHE35 }\end{array}$ \\
\hline $4 b$ & $\begin{array}{l}\text { 4-((8-hydroxyquinolin-5- } \\
\text { yl) diazenyl) -N- (5-methyl } \\
\text { isoxazol-3-yl) benzene } \\
\text { Sulphonamide }\end{array}$ & & -10.652 & $\begin{array}{l}\text { PHE200, VAL295, LEU391, } \\
\text { PHE395, LEU408, TYR409, VAL444 }\end{array}$ \\
\hline $4 c$ & $\begin{array}{c}\text { 2-hydroxy-5- } \\
\text { ((4-(N-(5-methylisoxazol-3-yl) } \\
\text { sulfamoyl) phenyl) diazenyl) } \\
\text { benzoic acid }\end{array}$ & & -11.386 & $\begin{array}{l}\text { MET196, PHE200, GLN203, } \\
\text { VAL291, PHE292, LEU294, } \\
\text { VAL295, LEU298, LEU391, } \\
\text { PHE395, LEU408 }\end{array}$ \\
\hline $4 d$ & $\begin{array}{c}\text { 4-((2-hydroxynaphthalen- } \\
\text { 1-yl) diazenyl)-N-(5- } \\
\text { methylisoxazol-3-yl) benzene } \\
\text { sulfonamide }\end{array}$ & & -12.980 & $\begin{array}{c}\text { LYS83, VAL89, LEU93, ILE112, } \\
\text { TYR115, VAL116, SER119, } \\
\text { ARG120, TYR122 }\end{array}$ \\
\hline $4 e$ & $\begin{array}{l}\text { 4-((3-formyl-4-hydroxyphenyl) } \\
\text { diazenyl)-N-(5- } \\
\text { methylisoxazol-3-yl) benzene } \\
\text { sulfonamide }\end{array}$ & & -11.159 & $\begin{array}{l}\text { MET196, PHE200, GLN203, } \\
\text { VAL291, LEU294, VAL295, LEU298, } \\
\text { HIS388, LEU391, PHE395, LEU408 }\end{array}$ \\
\hline $4 f$ & $\begin{array}{l}\text { 4-((4-hydroxy-5-isopropyl- } \\
\text { 2-methylphenyl)diazenyl)- } \\
\text { N-(5-methylisoxazol-3-yl) } \\
\text { benzenesulphonamide }\end{array}$ & & -12.386 & $\begin{array}{c}\text { PHE200, GLN208, VAL295, } \\
\text { LEU391, PHE395, PHE404, } \\
\text { LYS405, PHE407, LEU408, TYR409, } \\
\text { VAL444, VAL447 }\end{array}$ \\
\hline $\begin{array}{l}\text { Acet y l } \\
\text { salicylic } \\
\text { acid }\end{array}$ & 2-acetoxybenzoic acid & & -10.256 & $\begin{array}{c}\text { PHE210, ASN375, ILE377, ALA378, } \\
\text { PHE381 }\end{array}$ \\
\hline
\end{tabular}
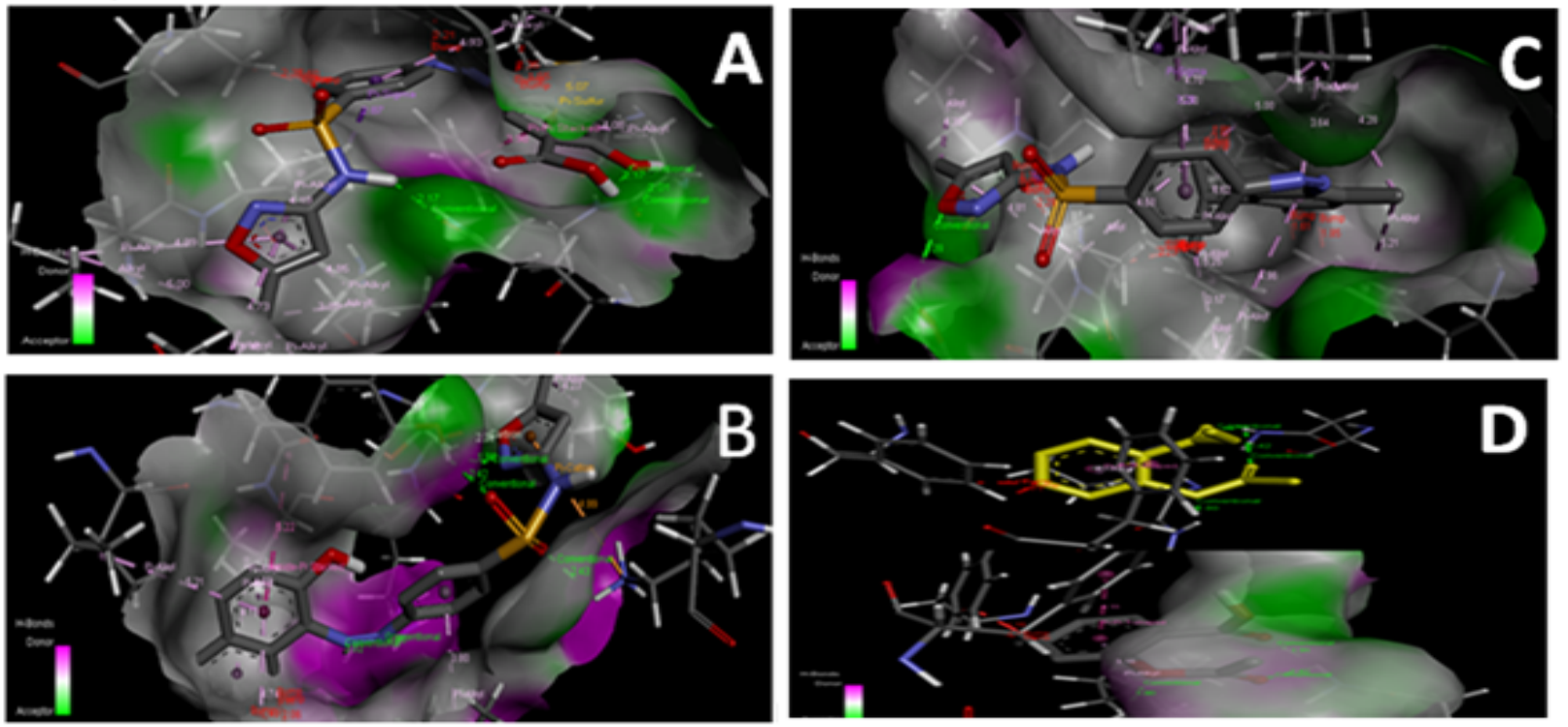

Fig. 3: Docking images captured by the software discovery studio visualizer

Docking images captured during interaction of $1 \mathrm{CX} 2$ with SMZ derived molecules A. 4c, B. 4d, C. 4f and D. standard acetylsalicylic acid, respectively

highest binding affinity to COX-2 and compared to standard those of ASA at $-10.256 \mathrm{kcal} / \mathrm{mol}$. Compound 4-((2-hydroxynaphthalen-1-yl)diazenyl)$\mathrm{N}$-(5-methylisoxazol-3-yl)benzene sulphonamide (4d) binds to several amino acids, LYS83, VAL89, LEU93,
ILE112, TYR115, VAL116, SER119, ARG120 and TYR122 of the active site of COX-2.

The absorption spectra of the molecule 4-((4-hydroxy-2oxo-2H-chromen-3-yl)diazenyl)-N-(5-methylisoxazol3-yl)benzene sulphonamide (4a) gave the largest 
bathochromic shift $425 \mathrm{~nm}$ with isopropanol in comparison to other solvents. Molecule $4 \mathrm{a}$ and $4 \mathrm{c}$ showed the maximum wave length $\left(\lambda_{\max }\right)$ with ethanol at 420 and $370 \mathrm{~nm}$, respectively; the molecule $4 \mathrm{~b}$, $4 \mathrm{~d}$ and $4 \mathrm{e}$ showed at 403.8, 480 and $360 \mathrm{~nm}$ with methanol, respectively, while the molecule $4 \mathrm{f}$ showed $\lambda$ ${ }_{\max }$ at $409 \mathrm{~nm}$ with1 4 -dioxane. The molecule $4 \mathrm{~d}$ showed maximum bathochromic shift with methanol, ethanol, DMSO and THF in comparison to other molecules.

Results of acute oral toxicity study indicated that the synthesized isoxazole derivatives were safe up to 2000 $\mathrm{mg} / \mathrm{kg}$ with no mortality, no toxic symptoms and no gross behavioural changes observed in Wistar rats. In the acetic acid-induced model in the control group, acetic acid produced $52.8 \pm 5.3$ writhes in $10 \mathrm{~min}$ of observation period and the standard ASA $(100 \mathrm{mg} /$ $\mathrm{kg}$ ) inhibited pain by $63.63 \%$ and compounds (4a-4f) produced $16.09,13.25,44.88,58.33,36.553$ and 57.76 $\%$ pain inhibition at $50 \mathrm{mg} / \mathrm{kg}$ dose level. Among which the 2-naphthol and thymol coupled diazotized isoxazole molecules $4 \mathrm{~d}$ and $4 \mathrm{f}$ reported with significant percent of pain inhibition 58.33 and $57.76 \%$ respectively at a dose of $50 \mathrm{mg} / \mathrm{kg}$ body weight. In radiant heat model, the negative control group of animal showed $4.03 \pm 0.18$ of reaction time by the end of $60 \mathrm{~min}$, while the compound $4 \mathrm{~d}$ showed significant analgesic activity by the end of 120 and $180 \mathrm{~min}$ for a reaction time of $6.7 \pm 0.59$ and $6.3 \pm 0.5$. The compound $4 \mathrm{f}$ noticed with reaction time of $5.8 \pm 0.52$ and $5.7 \pm 0.5$ by the end of 120 and $180 \mathrm{~min}$, respectively. The maximum percent inhibition of pain produced by the test compounds at a dose of $50 \mathrm{mg} /$ $\mathrm{kg}$ were for compound $4 \mathrm{~d}(80), 4 \mathrm{f}(62.85), 4 \mathrm{e}(51.42)$ and $4 \mathrm{a}$ (48.57) and for the standard (77.14). However, in both acetic acid-induced writhing and radiant heat models molecules $4 \mathrm{~d}$ and 4 f exerted significant analgesic activity. The molecules $4 \mathrm{~d}$ and $4 \mathrm{f}$ significantly $(\mathrm{p}<0.05)$ inhibited the inflammatory oedema when compared to negative control by the end $120 \mathrm{~min}$ of carrageenan injection.
The antioxidant activity of the isoxazole derivatives was investigated using the DPPH assay and the results were compared with those of standard BHT. The $\mathrm{IC}_{50}$ values of 4-[(4-hydroxy-2-oxo-2H-chromen-3-yl)diazenyl]$\mathrm{N}$-(5-methylisoxazol-3-yl)benzene sulphonamide (4a), 4-((8-hydroxyquinolin-5-yl)diazenyl)-N(5-methylisoxazol-3-yl)benzene sulphonamide (4b), 2-hydroxy-5-((4-(N-(5-methylisoxazol-3yl)sulfamoyl)phenyl)diazenyl)benzoic acid (4c), 4-((2-hydroxynaphthalen-1-yl)diazenyl)-N-(5methylisoxazol-3-yl)benzene sulphonamide (4d), 4-((3-formyl-4-hydroxyphenyl)diazenyl)-N-(5methylisoxazol-3-yl)benzene sulphonamide (4e), 4-((4-hydroxy-5-isopropyl-2-methylphenyl)diazenyl)$\mathrm{N}$-(5-methylisoxazol-3-yl)benzene sulphonamide (4f) and the standard BHT were, $30 \pm 0.78,52 \pm 1.13$, $59.7 \pm 0.03,38 \pm 1.4,48 \pm 0.89,40 \pm 0.59$ and $31 \pm 0.70 \mu \mathrm{g} /$ $\mu 1$, respectively. However, compound (4a) exhibited $\mathrm{IC}_{50}$ at the lowest concentration level of $30 \pm 0.78 \mu \mathrm{g} / \mu \mathrm{l}$ in comparison to other compounds tested. The molecule $4 \mathrm{~d}$ and $4 \mathrm{f}$ showed significant antioxidant activity at 10 and $50 \mu \mathrm{g} / \mu \mathrm{l}$, with \% inhibitions of $36.53 \pm 0.02$ and $61.41 \pm 0.04$ when compared to the standard.

Compounds $4 \mathrm{a}$ and $4 \mathrm{e}$ showed significant antimicrobial activity against $S$. aureus $_{(\text {res })}$ and $C$. neoformans in comparison to amoxicillin and fluconazole, respectively, whereas compounds $4 \mathrm{~b}-4 \mathrm{~d}$ showed significant antibacterial activity against $E$. coli ${ }_{(r e s)}$ in comparison to standard (Table 3 ). The inhibitory property of the isoxazole derivatives was determined in the concentration range of $500-31.25 \mu \mathrm{g} / \mathrm{ml}$ to find out the MIC values in $\mu \mathrm{g} / \mathrm{ml}$. The compound $4 \mathrm{a}$ have been exhibited more potential antibacterial activity by inhibiting the growth of $S$. aureus $_{(\text {res })}, C$. Albicans and C. neoformans at a concentration level of 31.25 $\mu \mathrm{g} / \mathrm{ml}$. The reported MIC results against different microbial strains by the compound $4 \mathrm{~b}$ would be considered as highly effective antimicrobial compound

TABLE 3: ANTIMICROBIAL ACTIVITY OF SMZ ANALGUES 4A-4F AGAINST DIFFERENT MICROBIAL STRAINS

\begin{tabular}{lccccc}
\hline Comps. & E. coli $_{(\text {res })}$ & K. pneumonia & S. aureus & C. albicans & C. neoformans \\
\hline 4a & - & - & $24.00 \pm 0.63^{*}$ & $24.17 \pm 0.75$ & $31.50 \pm 0.84^{*}$ \\
4b & $20 \pm 2^{*}$ & $15.83 \pm 1.17$ & $25.33 \pm 1.97^{*}$ & $8.83 \pm 1.33$ & $17.5 \pm 2.17$ \\
4c & $22 \pm 1.55^{*}$ & $25 \pm 1.1^{*}$ & - & $21 \pm 2.83$ & $21.17 \pm 0.98$ \\
4d & $15.67 \pm 1.21^{*}$ & $13 \pm 2.19$ & $15.17 \pm 2.23$ & - & $14.17 \pm 0.75$ \\
4e & - & - & $28.17 \pm 1.47^{*}$ & - & $18.17 \pm 2.32^{*}$ \\
4f & $9.83 \pm 1.84$ & 9.57 & 9.41 & - & $7.83 \pm 1.17$ \\
RA/RA $^{1}$ & $12.67 \pm 1.51$ & $15.33 \pm 1.97$ & $13 \pm 1.67$ & $19.33 \pm 4.68$ & $24.17 \pm 1.94$ \\
\hline
\end{tabular}

Results expressed in mean \pm SD of zone of inhibitions in $\mathrm{mm}(\mathrm{n}=6)$, data were analyzed using One Way ANOVA followed by Dunnett's Post Hoc test, statistical significance at ${ }^{*} \mathrm{p}<0.05$ in comparison to the reference antibiotic (RA), - no zone of inhibition, $E$. coli ${ }_{\text {res) }}$ - Escherichia coli (resistant), K. pneumonia- Klebsiella pneumonia, S. aureus ${ }_{(\text {res })}$ - Staphylococcus aureus (resistant), C. albicans- Candida albicans ,C. neoformans- Cryptococcus neoformans 
when compared to other synthesized isoxazole derivatives. However, all the synthesized molecules showed potential fungal inhibitory property against C. neoformans.

The resultant docking score of the molecules suggested that the molecules $4 \mathrm{~d}$ and $4 \mathrm{f}$ would be potent COX2 inhibitors. It was also found that from the in vivo evaluations that the molecule $4 \mathrm{~d}$ and $4 \mathrm{f}$ showed highest analgesic and antiinflammatory activity among all compounds tested; its action could be due to linking 5-methylisoxazolyl moiety to 2-naphthol and thymol, respectively. Literature supports that isoxazole derived molecules have shown to possess the inhibitory property ofCOX-2 and also revealed that the nitrogen heterocyclic molecules bearing $-\mathrm{N}=\mathrm{N}$ - are responsible for inhibition of $\mathrm{COX}^{[14]}$. In present study, the synthesized molecules contained diazenyl function group along with isoxazole nucleus and sulphanilamide together in their structures. Overall structural activity relationships (SAR) study of all analogues had suggested that the presence of phenolic/enolic hydroxyl, diazenyl group and nitrogen containing heterocyclic rings in their structures could be responsible for exhibiting the antioxidant, antimicrobial and antioxidant property.

A series of isoxazolyl derivatives were prepared by azo-coupling reactions and evaluated to investigate their various biological actions. The result suggested that the analogue $4 \mathrm{~d}$ and $4 \mathrm{f}$ exhibited significant analgesic, antioxidant and antimicrobial activities in comparison to standard drugs. Furthermore, the plausible binding sites of these synthesized derivatives could be designated through molecular docking. Thus, SARs of synthesized derivatives suggested that the presence of 5-methylisoxazolyl moiety and a phenolic system, could yield potential leads for developing new therapeutic agents.

\section{Acknowledgements:}

The authors thank the Dean, School of Pharmaceutical Sciences, Siksha 'O' Anusandhan University and Deputy Director RIPAES, Bhubaneswar, India.

\section{REFERENCES}

1. Wang XL, Wan K, Zhou CH. Synthesis of novel sulfanilamidederived 1,2,3-triazoles and their evaluation for antibacterial and antifungal activities. Eur J Med Chem 2010;45:4631-9.

2. Wilkinson BL, Bornaghi LF, Wright AD, Houston TA, Poulsen SA. Anti-Mycobacterial Activity of a Bis-Sulfonamide. Bioorganic Med Chem Lett 2007;17:1355-7.

3. SahooJ,Sahoo SK, Paidesetty SK. Biological evaluation of novel a-heteroaryl/arylazo 2-naphthol analogs and the transitional metal complexes derived from 4-((2-hydroxynaphthalen-1yl) diazenyl)-1,5-dimethyl-2-phenyl-1h-pyrazol-3(2H)-one. Indian Drugs 2016;53:15-24.

4. Anuradha RC, Bhanudas SK, Swati CJ, Pradeep DO, Vasant VC, Suresh US, et al. Synthesis and evaluation of analgesic, antiasthmatic activity of (E)-1-(8-hydroxyquinolin-7-yl)-3phenylprop-2-en-1ones. Arab J Chem 2014;10:46.

5. Zimecki M, Bachor U, Maczynsk M. Isoxazole Derivatives as Regulators of Immune Functions. Molecules 2018;23:2724.

6. Patil A, Disouza J, Pawar S. Health benefits of probiotics by antioxidant activity: A review. Pharma Times 2018;50:9-11.

7. Sahoo J, Paidesetty SK. Antimicrobial, analgesic, antioxidant and in silico study of synthesized salicylic acid congeners and their structural interpretation. Egypt J Basic Appl Sci 2015;2:268-80.

8. Sahoo J, Paidesetty SK. Biological evaluation and spectral characterization of 4-hydroxy coumarin analogues. J Taibah Univ Med Sci 2015;10:306-19.

9. Sahoo J, Paidesetty SK. Study of antimicrobial, analgesic wound healing and antioxidant activities of some newly synthesized oxychinolin derivatives and their characterization. Beni-Suef Univ J Appl Sci 2015;4:232-45.

10. Sahoo J, Parween G, Sahoo S, Mekap SK, Sahoo S, Paidesetty SK. Synthesis, spectral characterization, in silico and in vitro antimicrobial investigations of some Schiff base metal complexes derived fromazo salicylaldehyde analogues. Ind J Chem 2016;55:1267-76.

11. Oruc EE, Kocyigit KB, Oral B, Altunbas-Tolku HZ, Kabasakal L, Rollas S. Synthesis of some novel azo derivatives of 3,5-dimethyl-1-(2-hydroxyethyl) pyrazole as potent analgesic agents. Arch Pharm 2006;339:267-72.

12. Jayanna ND, Vagdevi HM, Dharshan JC, Raghavendra R, Sandeep T. Synthesis, antimicrobial, analgesic activity, and molecular docking studies of novel1-(5,7-dichloro-1,3benzoxazol-2-yl)-3-phenyl-1H-pyrazole-4-carbaldehyde derivatives. Med Chem Res 2013;22:1-9.

13. Eddy NB, Leimback D. Synthetic analgesics II dithienylbutenyl and dithienylbutylamines. J Pharm Exp Ther 1953;107:385-93.

14. Shaikh A, Meshram JS. Design, synthesis and pharmacological assay of novel azo derivatives of dihydropyrimidinones. Cogent Chem 2015;1:1019809.

15. McFarland J. Standardization of bacterial culture for disc diffusion assay. J Am Med Assoc 1987;49:1176-1178. 